\title{
Oscillations of a magneto-sensitive elastic sphere
}

\author{
Holm Altenbach ${ }^{1, *}$, Igor A. Brigadnov ${ }^{2, * *}$, and Victor A. Eremeyev ${ }^{3, * * *}$ \\ ${ }^{1}$ Lehrstuhl für Technische Mechanik, Zentrum für Ingenieurwissenschaften, Martin-Luther-Universität Halle-Wittenberg, \\ 06099 Halle (Saale), Germany \\ ${ }^{2}$ Department of Computer Science, North-West State Technical University, Millionnaya Str. 5, 191186 St. Petersburg, \\ Russian Federation \\ ${ }^{3}$ South Scientific Center of RASci and South Federal University, Milchakova Str. 8a, 344090 Rostov on Don, Russian \\ Federation
}

Key words Magnetoelasticity, non-linear elasticity, magneto-sensitive elastomers, oscillations, Lyapunov function.

\begin{abstract}
Magneto-sensitive (MS) or magnetorheological (MR) elastomers are smart materials whose mechanical properties change instantly by the application of a magnetic field. MS elastomers are widely used in the practice as elements of MEMS (sensors, actuators, etc.), for example, in medical devices. The behavior of MS elastomers under time-dependent magnetic field is a complex process and till now is not investigated in all details. Here we present the statements of the dynamic boundary-value problem of a MS elastomer and demonstrate the special features of the dynamic behavior of such system. As an example both the nonlinear and the linear oscillations of a MS elastic sphere are considered. The control of radially symmetric oscillations of MS elastic sphere by a homogeneous magnetic field is investigated. The presented results can be used for control and generation of radially symmetric acoustic waves using magnetic field excitation.
\end{abstract}

\section{Introduction}

Magneto-sensitive (in the literature so-called magnetorheological - MR) elastomers are materials that respond to an applied magnetic field with an instantaneous change in the mechanical behavior. An improved understanding of MS elastomers is demanded by the prospect to provide simple, reliable and rapid-response interfaces between control laws and mechanical systems. It is well recognized that MS elastomers have the potential to improve the design of electromechanical devices and their operations. For example, an elastomer with field dependent properties may be used as a device with a variable stiffness.

The magnetic efficiency to change field-dependent mechanical properties is optimized by choosing a particle material with high magnetic saturation. Cobalt has the largest saturation of $2.4 \mathrm{~T}$ of all known elements, however it is not used commercially. In general, an alloy of iron with a magnetic saturation of $2.1 \mathrm{~T}$ is used as an additive in the mixing process of MS elastomeric compounds.

Magneto-sensitive solid elastomers are less used in comparison to their electro-sensitive (ES) counterparts. Both elastomers are composed of polarizable particles, dispersed in a polymer medium, having a size of the order of a few microns (typically from $10^{-7}$ to $10^{-5} \mathrm{~m}$ ). Carrier fillers are selected based upon their electro-magnetic and thermo-mechanical properties: silicone and/or other rubber-like materials with a very small electric conductivity. The typical particle volume fraction is between 0.1 and 0.5. During the manufacturing process of MS elastomers, the isotropy condition inherent of the filler material is maintained in the final composite. Therefore, these materials are considered isotropic and non-conductive. However, MS elastomers become non-homogeneous due to the presence and distribution of particles in the carrier filler.

Magneto-sensitive materials are introduced in the late 1940s [42]. At the same time, initial experimental results on electrorheological fluids where published by Winslow [54]. Following the research of these materials, the major interest was dedicated towards ES materials in the late 1940s and early 1950s, which is evident by the large number of patents and

* $\quad$ E-mail: holm.altenbach@iw.uni-halle.de

** Corresponding author, E-mail: brigadnov@mail.ru,

*** E - mail: eremeyev@math.rsu.ru 
publications. Except the interest immediately following Rabinow's work, there has been little new information about and publications on MS media. Recently however, MS elastomers have been recognized as a commercially viable product. Therefore, this wide range of potential applications and associated economic benefits are the reason for the intense research on these materials in recent years, see, for example [1,3-6, 9-12, 24-26, 28-32, 35, 44, 47, 51-53]. A number of MS elastomers and various MS elastomer-based systems have successfully been brought into the market, including adaptive tuned vibration absorbers, sensors, actuators, medicine devices, stiffness tunable mounts, suspensions, and automotive bushing.

Structurally, field responsive elastomers can be thought of as the solid analogs of field responsive non-colloidal suspensions or fluids, see for example [28]. There are however some distinct differences in the way in which these two classes of materials are intended to be used. The most important one is that the field sensitive particles within the elastomer composite are intended to always operate in the pre-yield regime, see [10]. Therefore, field responsive elastomers can best be described by a field dependent modulus, see for example [6, 14,31,32,44].

Non-linear theory of elasticity of magneto-elastomers was developed in [7, 8, 14-17, 20, 21, 33]. The similar problems for the electro-elastomers were considered in $[18,19,43]$. Mechanics of thin-walled magneto-elastic structures like a membranes was considered in [2,46]. Example of practical using of magneto-elastic membranes is given in [37]. Magnetoelastomers and electro-elastomers are special examples of electro-magneto-sensitive materials. For full details of the general theory of electrodynamics of continua, we refer to, for example, [13, 22, 23, 40, 41].

In the paper we consider the spherically symmetric deformation of an isotropic elastic MS sphere in a homogeneous magnetic field. Following [7,14-17], in Sect. 1 we recall the general statement of dynamics of magneto-sensitive elastomers. In Sects. 2 and 3 we investigate the oscillations of magneto-elastic sphere in the case of finite (nonlinear) and infinitesimal (linear) deformations, respectively. We present the appropriate differential equations of dynamics for sphere inflated by the inner pressure, where the magnetic field is an external parameter changing the stiffness of the sphere. For the case of nonlinear constitutive relation, in Sect. 2 we investigated oscillations of magneto-sensitive sphere. Appropriate phase portraits are presented and analyzed. In Sect. 3 for the linear Hook's constitutive relation the problem of the parametric control and damping of oscillations for the appropriate linear dynamical system is solved by the Lyapunov method [27]. The presented theoretical results are based on the theorem about asymptotic stability in reference to the part of variables [45] and the Barbashin-Krasovski theorem [36].

\section{Basic equations of incompressible magneto-elastomers}

Following [7,14-17], let us recall the basic relations of the theory of finite magneto-elasticity. For definiteness we consider an incompressible material in the absence of external body forces. The motion of the body is described by the positionvector in the actual configuration $x$

$$
x=x(X, t),
$$

while $X$ is the position-vector in the reference configuration. We use standard notations

$$
F=\left(\nabla_{X} x\right)^{\mathrm{T}}, \quad J=\operatorname{det} F,
$$

where $\boldsymbol{F}$ is the gradient of the position-vector, $\nabla_{X}$ is the nabla operator with respect to $X$. For rubber-like materials we use the incompressibility condition

$$
J=1
$$

The constitutive equations of an incompressible isotropic magneto-elastic solid are given by

$$
\begin{aligned}
& W=W\left(I_{1}, I_{2}, I_{4}, I_{5}, I_{6}\right), \\
& \sigma=-p \boldsymbol{I}+F \cdot \frac{\partial W}{\partial F}, \\
& M_{\mathrm{e}}=-\frac{\partial W}{\partial B},
\end{aligned}
$$

where $W$ is the specific free energy given as a function of the following set of invariants $[7,15,17]$

$$
I_{1}=\operatorname{tr} b, \quad I_{2}=\frac{1}{2}\left[(\operatorname{tr} b)^{2}-\operatorname{tr} b^{2}\right], \quad I_{4}=B^{2} \equiv|B|^{2}, \quad I_{5}=(\boldsymbol{b} \cdot B) \cdot B, \quad I_{6}=\left(\boldsymbol{b}^{2} \cdot \boldsymbol{B}\right) \cdot B,
$$

$b=F \cdot F^{T}$ is the left Cauchy-Green deformation tensor, $B$ is the vector of magnetic induction, $\sigma$ is the Cauchy stress tensor, $M_{\mathrm{e}}$ is the normalized vector of magnetization (see [15]), $p$ is a Lagrange multiplier associated with the constraint (2), and $I$ is the second-order identity tensor. From the physical point of view $p$ is the hydrostatic pressure [39]. 
The equation of motion and the field equation have the following form

$$
\begin{aligned}
& \nabla_{x} \cdot \sigma+\left(\nabla_{x} B\right) \cdot M_{\mathrm{e}}=\rho \dot{\boldsymbol{v}} \\
& \nabla_{x} \cdot B=0
\end{aligned}
$$

where $\nabla_{x}$ is the nabla operator in the actual configuration, $\rho$ is the density, $v=\dot{x}$ is the velocity, $(.$.$) is the material$ derivative with respect to the time $t$. Further we assume that $B$ is homogeneous and depends only on $t$. The assumption $B=B(t)$ means that there is no backward influence of the oscillating sphere on the surrounding magnetic field. Then Eqs. (4) reduce to the standard one

$$
\nabla_{x} \cdot \sigma=\rho \dot{v}
$$

The static boundary conditions have the standard form too

$$
\sigma \cdot n=f
$$

where $n$ is a unit outer normal to the boundary of the body and $f$ is an external surface load.

Let us specify the form of the energy given by

$$
W=\frac{1}{2} \mu\left(I_{4}\right)\left(I_{1}-3\right)
$$

with $\mu\left(I_{4}\right)=\mu_{0}\left(1+\eta I_{4}\right), \eta>0$. For small deformations $\mu_{0}$ is the shear modulus in the absence of the magnetic field, $\eta$ describes the influence of the magnetic field on the shear modulus. Equation (7) is the classical neo-Hookean model, which is widely used in the mechanics of elastomers (see, for example, $[38,48]$ ) with an elastic modulus highly depending on the magnetic field induction intensity. More general constitutive equations were considered, for instance, in [7, 15, 17]. Using (7) we obtain

$$
\sigma=-p \boldsymbol{I}+\mu\left(I_{4}\right) b .
$$

Thus, the boundary-value problem (5), (6) describes the deformations of MS elastomers under action of both the external forces and the magnetic field. Let us note that Eqs. (5), (6) contain $B(t)$ only as a parameter. On the other hand, the dependence $B$ on $t$ posses one to generate and control the vibrations of MS elastomer based devices. To illustrate this idea we consider an one-dimensional problem for MS elastomers in the next section.

\section{Nonlinear oscillations of a magneto-elastic sphere}

Let us consider the oscillations of a hollow magneto-elastic sphere under action of a homogenous magnetic field $B=\boldsymbol{B}(t)$ and a inner hydrostatic pressure $\tilde{p}$. In the reference configuration the sphere has the inner and the outer radii $r_{0}$ and $r_{1}$, respectively. In the spherically symmetric case the position-vector is given by

$$
x=R(r, t) e_{r}
$$

where $r \in\left[r_{0}, r_{1}\right]$ is the radial component of the spherical Lagrangian coordinates and $\boldsymbol{e}_{r}$ is the appropriate base vector (see, e.g. [38,39]), $R$ is an unknown function. In the actual configuration the inner and the outer radii are $R_{0}=R\left(r_{0}, t\right)$ and $R_{1}=R\left(r_{1}, t\right)$, respectively.

From (2) we immediately find that

$$
R(r, t)=\left(r^{3}+x(t)\right)^{1 / 3},
$$

where $x(t)$ is a new unknown function. Thus, $R_{1}=\left(r_{1}^{3}+x(t)\right)^{1 / 3}, R_{0}=\left(r_{0}^{3}+x(t)\right)^{1 / 3}$. Equation (9) is one of the well-known so-called universal solutions for incompressible solids (see, e.g. [38,50]. For magneto-elastomers the universal solutions were studied in [20]. From (9) it follows that the volume of the sphere is constant, i.e.

$$
R_{1}^{3}-R_{0}^{3}=r_{1}^{3}-r_{0}^{3}
$$

For the universal solution (9) Eq. (5) is satisfied identically by choosing of function $p=p(r, t)$.

For spherically symmetric deformations the boundary conditions are given by

$$
\sigma_{R R}\left(R_{1}, t\right)=0, \quad \sigma_{R R}\left(R_{0}, t\right)=-\tilde{p},
$$


where $\sigma_{R R}=e_{r} \cdot \sigma \cdot \boldsymbol{e}_{r}$. For brevity, we omitted the awkward computations, see, for details, [38], p. 348. Finally, Eqs. (5), (11) can be reduced to the ordinary differential equation (ODE) with respect to $x(t)$

$$
\rho\left[\ddot{x} \frac{R_{1}-R_{0}}{R_{1} R_{0}}-\frac{1}{6} \dot{x}^{2} \frac{R_{1}^{4}-R_{0}^{4}}{R_{1}^{4} R_{0}^{4}}\right]+12 x \int_{r_{0}}^{r_{1}} \frac{R^{3}+r^{3}}{R^{7}}\left(\frac{\partial W}{\partial I_{1}}+\frac{R^{2}}{r^{2}} \frac{\partial W}{\partial I_{2}}\right) \mathrm{d} r=3 \tilde{p} .
$$

Using (7) Eq. (12) is reduced to

$$
\alpha(x) \ddot{x}-\beta(x) \dot{x}^{2}+\mu \gamma(x) x=\tilde{p},
$$

where

$$
\alpha(x)=\frac{1}{3} \rho \frac{R_{1}-R_{0}}{R_{1} R_{0}}>0, \quad \beta(x)=\frac{1}{18} \rho \frac{R_{1}^{4}-R_{0}^{4}}{R_{1}^{4} R_{0}^{4}}>0,
$$

and

$$
\gamma(x)=4 \int_{r_{0}}^{r_{1}} \frac{x+2 r^{3}}{\left(x+r^{3}\right)^{7 / 3}} \mathrm{~d} r
$$

are essentially nonlinear functions, $\gamma(x)$ cannot be expressed in elementary functions. Let us note that under assumptions of incompressibility, $B=B(t)$, spherical symmetry and constitutive equations (7), we reduce exactly the system with infinite number of degrees of freedom to a mechanical system with one degree of freedom. The analysis of the general case may be performed applying more complex methods (see, for example, [34,49]). Even for the considered simple example we show that the system with one degree of freedom presented by Eq. (13) behaves similar as the system with infinite number of degrees of freedom.

Equation (13) is essentially nonlinear non-autonomous ODE with respect to $x(t)$ which can be solved only numerically. Let us assume a sinusoidal behavior of $B: B(t)=B_{0} \sin \omega t$, where $B_{0}$ is the magnitude, while $\omega$ is the frequency. Example of numerical simulations is presented in Figs. 1, 2. In Fig. 1 the phase portrait of (13) in the absence of magnetic field $(\boldsymbol{B}=0)$ is shown. Here the following dimensionless parameters $\bar{r}_{0}=0.9, \bar{r}_{1}=1\left(\bar{r}=r / r_{1}\right), \bar{p} \equiv \tilde{p} / \mu=0.01$ were used, and we keep notation $x$ for new dimensionless variable $x / r_{1}^{3}$. Two closed trajectories correspond to the initial data $x(0)=0.01, \dot{x}(0)=0$, and $x(0)=0.1, \dot{x}(0)=0$, respectively. In Fig. 2 two trajectories correspond to initial data $x(0)=0.01, \dot{x}(0)=0$, and $x(0)=0.1, \dot{x}(0)=0$, respectively. Here $\bar{\eta}=\eta B_{0}^{2}$ and $\bar{\omega}=\omega T, T=\sqrt{\rho / \mu_{0}}$, and the time interval is $[0,300 T]$. The trajectories of (13) can demonstrate complex behavior. For the case of low values of $\bar{\eta}$ describing the dependence of the shear modulus on $B$ one can see the weak influence on the oscillations (see top row in Fig. 2). In this case we have the behavior similar to Fig. 1. The middle and bottom rows in Fig. 2 demonstrate more complex behavior for small frequencies, one can see some type of instabilities. The increase of the frequency of $B$ leads to the stabilization of oscillations near the solutions with constant $\boldsymbol{B}$ similar to the behavior shown in Fig. 1. It means that using the external magnetic field we can "control" in some sense the motion of the sphere.

Further strict mathematical analysis of the oscillations on MS elastic sphere may be proved using additional assumption. In the next section we assume that the deformations are small.

\section{Oscillations of a linear elastic sphere}

We consider an isotropic linear elastic sphere of the inner radius $r_{0}$ and outer radius $r_{1}$ in the reference configuration. The spherically symmetric mapping is described by

$$
x(t, r)=X(r)+\boldsymbol{u}, \quad \boldsymbol{u}=U(t, r) e_{r},
$$

where $x$ and $X$ are again the actual and reference position-vectors as functions of the time $t$ and the radius $r$ only, $U$ is the field of the full radial displacements of the sphere. We assume that $U(t, r)=R_{0}(t)-r_{0}+u(t, r)$, where $u$ is the relative field of radial displacements such that $u\left(t, r_{0}\right) \equiv 0$.

For the case of small deformations we have no need to distinguish the Lagrangian and Eulerian coordinates, etc. The incompressibility condition reduces to $\nabla \cdot u=0$. Using latter relation in the case of radially symmetric deformation with the condition $u\left(t, r_{0}\right) \equiv 0$, we obtain

$$
U(t, r)=\left(R_{0}(t)-r_{0}\right)\left(\frac{r_{0}}{r}\right)^{2}
$$




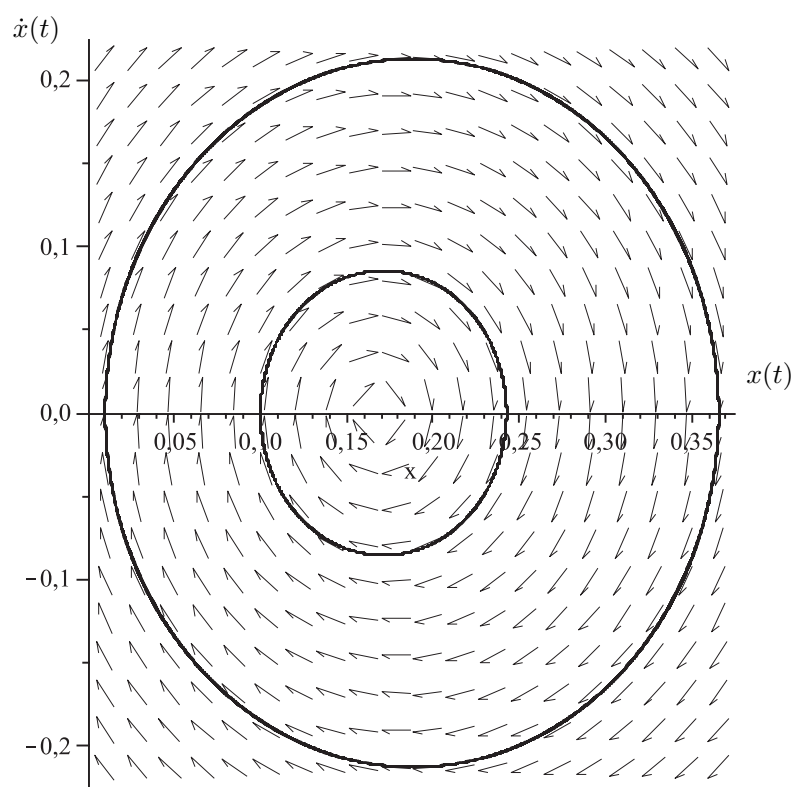

Fig. 1 Phase portrait of (13) in the absence of magnetic field.

For brevity, further we use the notation $a(t)=R_{0}(t)$.

In the considered problem the full displacements are finite but deformations are small, therefore, for description of the actual configuration we can use the linear elasticity theory. As a result, for the Cauchy strain tensor having in the spherical coordinates the form

$$
\varepsilon(t, r)=\left(a(t)-r_{0}\right) \frac{r_{0}^{2}}{r^{3}}\left(-2 \boldsymbol{e}_{r} \boldsymbol{e}_{r}+\boldsymbol{e}_{\theta} \boldsymbol{e}_{\theta}+e_{\phi} \boldsymbol{e}_{\phi}\right),
$$

and the Cauchy stress tensor $\sigma$, the Hooke's law for incompressible materials is valid

$$
\sigma=\frac{2}{3} E \varepsilon-p I
$$

where $E$ is the Young modulus, $p$ is the hydrostatic pressure.

Neglecting the weight of the sphere, in a homogeneous magnetic field the equilibrium equation has the classical form

$$
\rho \ddot{\boldsymbol{x}}=\nabla \cdot \sigma .
$$

Substituting relations (14), (15) into Eq. (16) we obtain the condition $\sigma_{\theta \theta}=\sigma_{\phi \phi}$ and the differential equation for functions $a(t)$ and $p(t, r)$

$$
\rho_{0} \ddot{a}\left(\frac{r_{0}}{r}\right)^{2}+\frac{\partial p}{\partial r}=0
$$

which is true for every radius $r \in\left(r_{0}, r_{1}\right)$ and time $t>0$.

On the inner surface of the sphere we have the surplus pressure $\tilde{p}>0$ and the outside surface is free, therefore, the following boundary conditions are true

$$
\sigma_{R R}\left(r_{0}, t\right)=-\tilde{p}, \quad \sigma_{R R}\left(r_{1}, t\right)=0 .
$$

After integration of Eq. (17) in $r$ and taking into account the boundary conditions (18) we obtain a differential equation for the current inside radius of the sphere

$$
\ddot{a}+c\left(a-r_{0}\right)=f,
$$

where $c$ and $f$ are the effective stiffness and the external force, respectively. They have the following form

$$
c=\frac{4}{3}\left(\frac{E}{\rho_{0}}\right) \frac{D_{0}}{r_{0}^{2} r_{1}^{2}\left(r_{1}-r_{0}\right)}>0, \quad f=\left(\frac{\tilde{p}}{\rho}\right) \frac{r_{1}}{r_{0}\left(r_{1}-r_{0}\right)}>0, \quad D_{0}=r_{1}^{3}-r_{0}^{3} .
$$




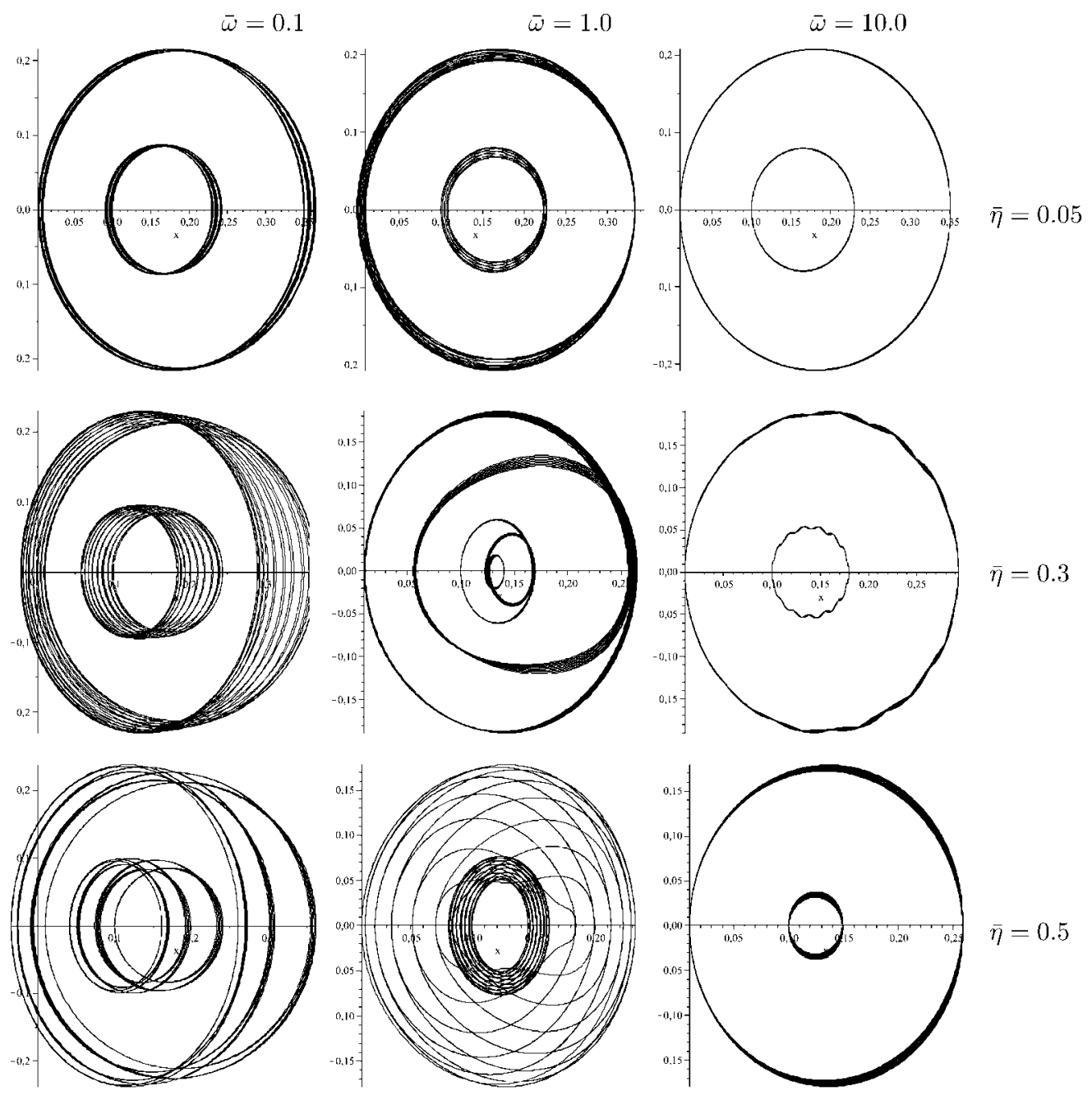

Fig. 2 Examples of trajectories of (13) for different parameters $\bar{\eta}$ and $\bar{\omega}$.

For a constant internal pressure $\tilde{p}_{0}>0$ the inflated sphere has the inner and the outer radii

$$
a_{*}=r_{0}\left[1+\frac{3}{4}\left(\frac{\tilde{p}_{0}}{E}\right) \frac{r_{1}^{3}}{D_{0}}\right]>r_{0}, \quad b_{*}=\left(a_{*}^{3}+D_{0}\right)^{1 / 3}>r_{1} .
$$

For example, the sphere with initial radii $r_{0}=0.1$ and $r_{1}=0.11 \mathrm{~m}$ prepared from the elastomer with the Young's modulus $E \approx 1.8 \mathrm{MPa}$ inflates to sizes $a_{*} \approx 0.15 \mathrm{~m}$ and $b_{*} \approx 0.155 \mathrm{~m}$.

It was proven [7] that in a magnetic field the Young's modulus of MS elastomers only increases according to the following relation

$$
E=E_{0}\left(1+\eta B^{2}\right)
$$

where $E_{0}$ is the Young modulus corresponding to zero magnetic flux density $B, \eta>0$ is the MS coefficient. From experiments it is well known that $\eta=\eta(\lambda)$, where $\lambda \in[0,0.5]$ is the MS particle volume fractions in an elastomer [10,35]. For commercially available MS elastomers $\eta(0)=0$ and $\eta(0.5) \approx 0.5$ [7].

For example, the examined above initially inflated sphere from a MS elastomer with $\lambda \approx 0.3$ in a homogeneous magnetic field $B \approx 0.8 \mathrm{~T}$ shrinks to sizes $R_{0} \approx 0.14 \mathrm{~m}$ and $R_{1} \approx 0.146 \mathrm{~m}$.

In the following section we will consider oscillations of a MS elastic sphere near the static equilibrium state (20). After the standard replacements $y=a-a_{*}$ and $\tau=\sqrt{c_{0}} t$, where $c_{0}$ is the the effective stiffness corresponding to zero magnetic field, Eq. (19) is transformed into the following simple form

$$
\ddot{y}+\left(1+\eta B^{2}\right) y=0 .
$$




\subsection{Control of oscillations}

Consider the problem of generation of harmonic oscillations with the desired frequency $\omega_{d}$ and amplitude $A_{d}$ in the system (22). The coefficient of the effective stiffness is given by the formula $c_{d}=\omega_{d}^{2}$. The multiplier with $y$ can be represented as

$$
1+\eta B^{2}=c_{d}+u_{g}
$$

where $u_{g}$ is the new control input. Let $B_{\max }$ be the magnetic saturation of the MS elastomer, typically $B_{\max }$ is about $1 \mathrm{~T}$ [7]. Then

$$
1<c_{d}<1+\eta B_{\max }^{2}
$$

or

$$
c_{d}=1
$$

The last conditions impose restrictions on the desired frequency. Introducing the function of energy

$$
E=\frac{1}{2}\left(\dot{y}^{2}+c_{d} y^{2}\right)
$$

and the desired energy

$$
E_{d}=\frac{1}{2} c_{d} A_{d}^{2}
$$

it is easy to check that

$$
\dot{E}=-y \dot{y} u .
$$

Let us consider the Lyapunov function

$$
V=\frac{1}{2}\left(E-E_{d}\right)^{2} .
$$

Then we can synthesize the control input from the condition of decreasing the Lyapunov function on the trajectories of the closed loop system [27]. Differentiating we get that $\dot{V}=-y \dot{y}\left(E-E_{d}\right) u$. Under the condition (24) let us choose as the control input

$$
u_{g}=F\left(y \dot{y}\left(E-E_{d}\right)\right),
$$

where $F(t)$ is a continuous, monotonously increasing function, $F(0)=0$. It may be bounded. It is reasonable to choose $F$ so, that

$$
1-c_{d}<F<1-c_{d}+\eta B_{\max }^{2}
$$

Under the condition (25) we choose as control

$$
u_{g}=F_{+}\left(y \dot{y}\left(E-E_{d}\right)\right),
$$

where $F_{+}(t)$ is a continuous function, $F_{+}(t)=0$ for $t \leq 0$, and it monotonously increases for positive $t$. It may be bounded.

For the deviation of energy the following equation holds true

$$
\left(E-E_{d}\right)=-\dot{y} y F\left(y \dot{y}\left(E-E_{d}\right)\right) .
$$

Proposition 3.1. The closed loop system (22), (23), (24) (or (25), (26), (27)) has the desired harmonic oscillation as the solution. The extended systems (22), (28) with conditions (23) and (24) (or (25)), and the control input in the form (26) or (27) are asymptotically stable with respect to the variable $E-E_{d}$. The closed loop system has also the equilibrium $y=0, \dot{y}=0$, which is unstable.

Proof. The asymptotic stability follows from the application of the theorem on asymptotic stability with respect to the part of variables [45] to the function $V$. Because $\dot{E} \geq 0$ in the small neighborhood of the point $(0,0)$ then this equilibrium is unstable in accordance to the Chetaev theorem on instability [36]. 


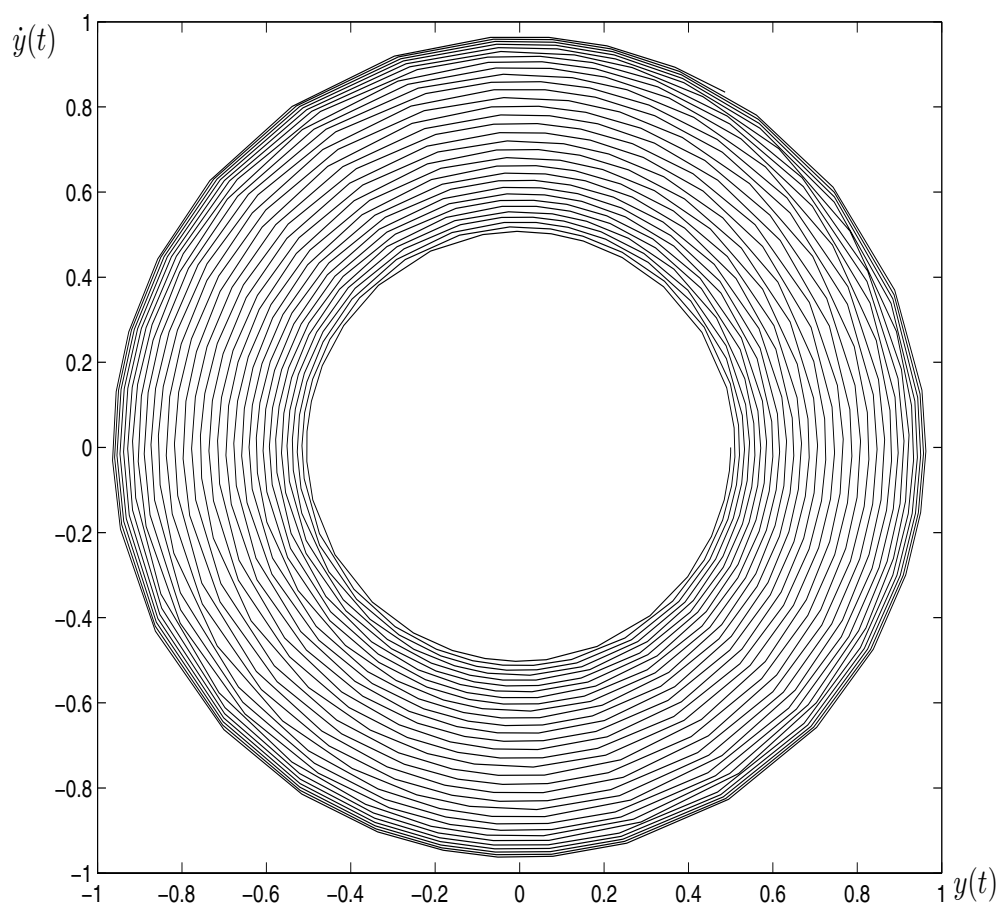

Fig. 3 Phase portrait of the closed loop system described in the Proposition 3.1 for the initial data $(0.5,0)$ in the time interval $[0,200]$

The example of phase portrait of the closed loop system described in the Proposition 3.1 is given in Fig. 3. Here we used dimensionless variables which are analogous to the ones used in the previous section.

Consider also the problem of oscillation damping. Construct the control input from the condition of decreasing $E$. Represent the multiplier with $y$ as

$$
1+\eta B^{2}=c+u_{0}
$$

where $c=1$ or $c>1$. In first case define as control input

$$
u_{0}=F_{+}(y \dot{y})
$$

and in the second case

$$
u_{0}=F(y \dot{y}),
$$

where the functions $F$ and $F_{+}$were mentioned above.

Proposition 3.2. The closed loop system (22), (29), (30) (or (31)) is globally asymptotic stable with respect to the variables $y, \dot{y}$.

Pro of. It follows from the application of the Barbashin-Krasovski theorem [36] to the function $E$.

We integrated numerically the closed loop system described in Proposition 3.1 with the following parameters: $c_{d}=1$, $A_{d}=1, F(p)=\arctan p / \pi$ on time interval $[0,100]$ with different initial data $\left(y_{0}, \dot{y}_{0}\right):(0.5,0),(0.8,-1),(1,1),(2,1)$, $(2,0)$. In addition, we integrated the system described in Proposition 3.2 on time interval $[0,200]$ with the parameter $c=1.5$ and different initial data $(1,0)$ and $(1,1)$. In all cases the desired convergence was observed.

\subsection{Non-stationary oscillations}

Rewrite the equation (22) as

$$
\ddot{y}+U y=0,
$$

where $U$ is a new control. Try to find the desired oscillations as $y_{d}(t)=A(t) \sin \left(\phi_{p}(t)\right)$, where $A(t), \phi_{p}(t)$ are sufficiently smooth functions. Differentiate and substitute in (32), then the coefficients with the harmonics equal to zero. We get the following differential equations

$$
2 \dot{A} \dot{\phi}_{p}+A \ddot{\phi}_{p}=0, \quad \ddot{A}-A \dot{\phi}_{p}^{2}+U A=0 .
$$


If we express the desired frequency as an exponential function of time $\phi_{p}=\kappa \exp (k t)+\mu,(\kappa, k \neq 0)$, then these differential equations have the following solutions

$$
U(t)=k^{2} \kappa^{2} t \exp (2 k t)-\frac{k^{2}}{4}, \quad A=c \exp \left(-\frac{k}{2} t\right), \quad c=\text { const. }
$$

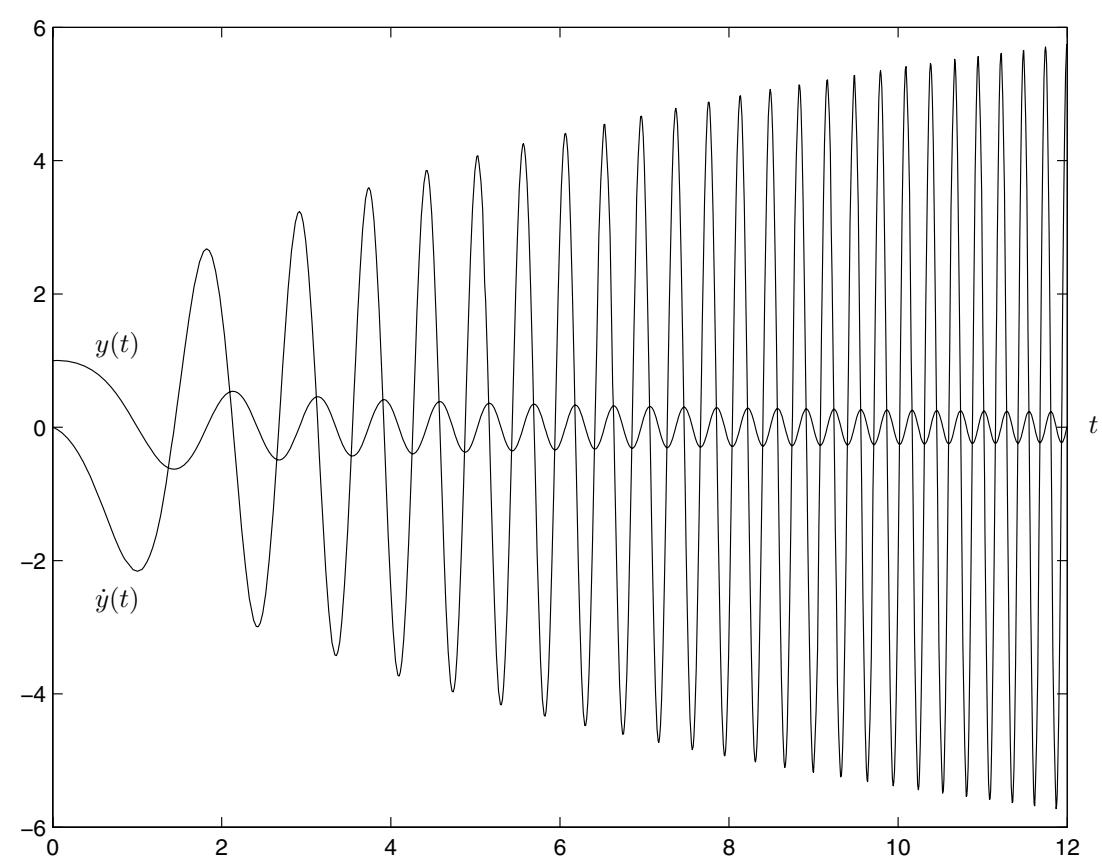

Fig. 4 Graphics of the functions $y(t)$ and $\dot{y}(t)$

In Fig. 4 there are the graphics of the functions $y(t), \dot{y}(t)$ for the given parameters $\kappa=1, k=1, \mu_{0}=1, y(0)=1$, and $\dot{y}(0)=0$ under the considered control.

\section{Conclusions}

In the paper the dynamic statement of the boundary-value problems of MS elastomers under homogeneous with respect to space but time-dependent magnetic field is given. The special property of the boundary-value problem is that the coefficients of the equations of motion may depend on time. As an example, we considered the radially symmetric oscillations of a MS incompressible elastic sphere. It was shown that using the external magnetic field one can generate and control the oscillations of the sphere. On the other hand, the considered system demonstrates complex behavior which highly depends on the type of external excitation. Such MS elastic sphere under internal pressure may be used, for example, as an actuator or working element of a microengine, based on MS elastomers.

Acknowledgements The research work was supported by DFG grant 436 RUS 17/21/07, by the DAAD program "Forschungsaufenthalte für Hochschullehrer und Wissenschaftler" in 2007, by the Russian Science Support Foundation and the Russian Foundation of Basic Research under grant 07-08-13589-ofi_c, and by the Presidium of Russian Academy of Sciences within the framework of the program 22.

\section{References}

[1] S. S. Abramchuk, D. A. Grishin, E. Yu. Kramarenko, G. V. Stepanov, and A. R. Khokhlov, Polymer Sci., Ser. A 48, 138-145 (2006).

[2] M. Barham, D. J. Steigmann, M. McElfresh, and R. E. Rudd, Acta Mechanica 191, 1-19 (2007).

[3] S. Bednarek, Appl. Phys. A 66, 643-650 (1998).

[4] S. Bednarek, Materials Science and Engineering B 55, 201-209 (1998).

[5] S. Bednarek, Appl. Phys. A 68, 63-67 (1999).

[6] L. Borcea and O. Bruno, J. Mech. Phys. Solids 49, 2877-2919 (2001). 
[7] I. A. Brigadnov and A. Dorfmann Int. J. Solids Struct. 40, 4659-4674 (2003).

[8] R. Bustamante, A. Dorfmann, and R. W. Ogden, J. Eng. Math. 59, 139-153 (2007).

[9] L. Chen, X. Gong, W. Jiang, J. Yao, H. Deng, and W. Li, J. Mater. Sci. 42, 5483-5489 (2007).

[10] J. D. Carlson and M. R. Jolly, Mechatronics 10, 555-569 (2000).

[11] F. Carpi, S. Galbiati, and A. Carpi, Biomedecine and Pharmacotherapy 60, 370-374 (2006).

[12] E. Coquelle, G. Bossis, D. Szabo, and F. Giulieri, J. Mater. Sci. 41, 5941-5953 (2006).

[13] A. DeSimone and P. Podio-Guidugli, Arch. Rational Mech. Anal. 136, 201-233 (1996).

[14] A. Dorfmann and I. A. Brigadnov, Comput. Mat. Sci. 29, 270-282 (2004).

[15] A. Dorfmann and R. W. Ogden, Acta Mechanica 167, 13-28 (2004).

[16] A. Dorfmann and R. W. Ogden, Q. J. Mech. Appl. Math. 57, 599-622 (2004).

[17] A. Dorfmann and R. W. Ogden, Z. angew. Math. Phys. 56, 718-745 (2005).

[18] A. Dorfmann and R. W. Ogden, Acta Mechanica 174, 167-183 (2005).

[19] A. Dorfmann and R. W. Ogden, J. Elast. 82, 99-127 (2006).

[20] A. Dorfmann, R. W. Ogden, and G. Saccomandi, Int. J. Non-Linear Mech. 39, 1699-1708 (2004).

[21] A. Dorfmann, R. W. Ogden, and G. Saccomandi, Int. J. Solids Struct. 42, 3700-3715 (2005).

[22] J. L. Ericksen, J. Elast. 87, 95-108 (2007).

[23] A. C. Eringen and G. A. Maugin, Electrodynamics of Continua (Springer, New York, 1990).

[24] M. Farshad and A. Benine, Polymer Testing 23, 347-353 (2004).

[25] M. Farshad and M. Le Roux, Polymer Testing 24, 163-168 (2005).

[26] G. Filipcsei, I. Csetneki, A. Szilágyi, and A. M. Zrínyi, Adv. Polym. Sci. 206, 137-189 (2007).

[27] A. L. Fradkov, Avtom. i Telemekh. 213-229 (1999).

[28] J. M. Ginder and L. C. Davis, Appl. Phys. Lett. 65, 3410-3412 (1994).

[29] X. L. Gong, X. Z. Zhang, and P. Q. Zhang, Polymer Testing 24, 669-676 (2005).

[30] Y. Hua, Y. L. Wanga, X. L. Gong, X. Q. Gong, X. Z. Zhang, W. Q. Jiang, P. Q. Zhang, and Z. Y. Chen, Polymer Testing 24, 324-329 (2005).

[31] M. R. Jolly, J. D. Carlson, and B. C. Muñoz, Smart Mater. Struct. 5, 607-614 (1996).

[32] M. R. Jolly, J. D. Carlson, B. C. Muñoz, and T. Bullions, J. Intell. Mater. Syst. Struct. 7, 613-622 (1996).

[33] S.V. Kankanala, and N. Triantafyllidis, J. Mech. Phys. Solids 52, 2869-2908 (2004).

[34] H.K. Khalil, Nonlinear Systems, 3rd ed., (Prentice Hall, Upper Saddle River, New Jersey, 2002).

[35] W. Kordonsky, J. Magnetism Magnetic Materials 122, 395-398 (1993).

[36] N. N. Krasovski, Control of Dynamical Systems (in Russian) (Nauka, Moscow, 1985).

[37] M. Khoo and C. Liu, Sensors and Actuators A 89, 259-266 (2001).

[38] A. I. Lurie, Non-linear Theory of Elasticity (North-Holland, Amsterdam, 1990).

[39] A. I. Lurie, Theory of Elasticity (Springer, Berlin et al. 2005).

[40] G. A. Maugin, Continuum Mechanics of Electromagnetic Solids (Elsevier Science Publishers, Oxford, 1988).

[41] Y. H. Pao, in: Mechanics Today, Vol. 4, edited by S. Nemat-Nasser (Pergamon Press, Oxford, 1978), pp. 209-306.

[42] J. Rabinow, AIEE Trans. 67, 1308-1315 (1948).

[43] K. R. Rajagopal and M. Růžička, Continuum Mech. Thermodyn. 13, 59-78 (2001).

[44] Z. Rigbi and L. Jilkén, J. Magnetism Magnetic Materials 37, 267-276 (1983).

[45] V. V. Rumyantsev and A. S. Oziraner, Stability and Stabilization in Reference to the Part of Variables (in Russian) (Moscow, Nauka, 1987).

[46] D. J. Steigmann, Int. J. Non-Linear Mech. 39, 1193-1216 (2004).

[47] G. V. Stepanov, S. S. Abramchuk, D. A. Grishin, L. V. Nikitin, E. Yu. Kramarenko, and A. R. Khokhlov, Polymer 48, 488-495 (2007).

[48] L. R. G. Treloar, The Physics of Rubber Elasticity, 3rd edn (Oxford University Press, Oxford, 1975).

[49] H. Troger and A. Steindl, Nonlinear Stability and Bifurcation Theory: An Introduction for Engineers and Applied Scientists (Springer, Wien, 1991).

[50] C. Truesdell and W. Noll, in: Handbuch der Physik, Vol. III/3, edited by S. Flügge (Springer, Berlin, Heidelberg, New York, 1965), pp. 1-602.

[51] Z. Varga, G. Filipcsei, and M. Zrínyi, Polymer 46, 7779-7787 (2005).

[52] Z. Varga, G. Filipcsei, and M. Zrínyi, Polymer 47, 227-233 (2006).

[53] Y. Wang, Y. Hu, L. Chen, X. Gong, W. Jiang, P. Zhang, and Z. Chen, Polymer Testing 25, 262-267 (2006).

[54] W. M. Winslow, J. Appl. Phys. 20, 1137-1140 (1949). 